\title{
From Path Integrals to Dynamical Algebras: A Macroscopic View of Quantum Physics
}

\author{
Detlev Buchholz ${ }^{1} \cdot$ Klaus Fredenhagen $^{2}$ (D)
}

Received: 4 September 2019 / Accepted: 24 April 2020 / Published online: 4 May 2020

(c) The Author(s) 2020

\begin{abstract}
The essence of the path integral method in quantum physics can be expressed in terms of two relations between unitary propagators, describing perturbations of the underlying system. They inherit the causal structure of the theory and its invariance properties under variations of the action. These relations determine a dynamical algebra of bounded operators which encodes all properties of the corresponding quantum theory. This novel approach is applied to non-relativistic particles, where quantum mechanics emerges from it. The method works also in interacting quantum field theories and sheds new light on the foundations of quantum physics.
\end{abstract}

Keywords Path integral · Arrow of time - Operator algebra - Quantization

\section{Introduction}

Path integrals [3, 4] are a standard tool in the theoretical description of quantum systems. Starting from a classical theory, describing paths (orbits) of the underlying system in configuration space and an action, governing its dynamics, they provide formulas for the propagators, i.e. the time ordered scattering operators in the resulting quantum theory. These formulas yield useful algorithms for the treatment of concrete problems. The proper definition of the underlying functional integrals is a quite subtle matter, however. It frequently requires reformulations of the integrals, e.g. in Euclidean space or on discrete lattices, which defy a direct physical interpretation. Moreover, changes of the states of the system, such as the passage from vacuum to thermal states, require adequate modifications of the integrals. And, last but not least, even though this approach is known to give satisfactory results, its conceptual foundations remained obscure.

Klaus Fredenhagen

fredenha@ mail.desy.de

1 Mathematisches Institut, Universität Göttingen, Bunsenstr. 3-5, 37073 Göttingen, Germany

2 II. Institut für Theoretische Physik, Universität Hamburg, Luruper Chaussee 149,

22761 Hamburg, Germany 
It is therefore gratifying that the essence of the path integral formalism can be replaced by simple algebraic relations without having to dive into the mathematical subtleties of functional integrals on infinite dimensional configuration spaces. Moreover, this reformulation sheds new light on the foundations of quantum physics. This new algebraic framework was established in quantum field theory in an effort to amend the axiomatic framework by some concrete dynamical input [1]. It was then extended to quantum mechanics, where its conceptual foundations were also settled [2]. It is the aim of the present letter to clarify its relation to the path integral formalism.

\section{Path Integrals}

Consider a system of $N$ distinguishable particles of equal mass (put equal to 1 ) whose orbits are given by functions of time $t \mapsto \boldsymbol{x}(t) \in \mathbb{R}^{3 N}$. Their unperturbed motion is fixed by the Lagrangean $\mathcal{L}_{0}(\dot{\boldsymbol{x}})=(1 / 2) \dot{\boldsymbol{x}}^{2}$. Temporary perturbations of this motion and interactions between the particles are described by time dependent potentials

$$
\boldsymbol{x} \mapsto V_{t}(\boldsymbol{x})=\sum_{k} g_{k}(t) V_{k}(\boldsymbol{x}),
$$

where the functions $g_{k}$ indicate when and for how long the potentials $V_{k}$ are effective. Their impact on any given orbit $\boldsymbol{x}$ is given by functionals $F[x]=\int_{t_{i}}^{t_{f}} d t V_{t}(\boldsymbol{x}(t))$, where $t_{i}$ is any time before the perturbation starts and $t_{f}$ any time after it has finished; the square bracket $[\boldsymbol{x}]$ indicates that a quantity depends on the whole orbit $\boldsymbol{x}$. Turning to the quantum system, the effect of the perturbations can be described by a unitary scattering matrix $S(F)$. It is given by the formula

$$
S(F)=e^{i t_{f} H_{0}} U_{F}\left(t_{f}, t_{i}\right) e^{-i t_{i} H_{0}},
$$

where $H_{0}$ is the Hamiltonian of the unperturbed system and $U_{F}\left(t_{f}, t_{i}\right)$ is the propagator for the perturbed Hamiltonian $H_{0}+V_{t}(\boldsymbol{Q}), \boldsymbol{Q}$ being the position operator. The kernel of the propagator is given by a path integral in position space, involving the above functional $F$,

$$
\left\langle\boldsymbol{q}\left|U_{F}\left(t_{f}, t_{i}\right)\right| \boldsymbol{q}^{\prime}\right\rangle=\int_{\boldsymbol{q}}^{\boldsymbol{q}^{\prime}} \mathscr{D} \boldsymbol{x} \exp \left(i \int_{t_{i}}^{t_{f}} d t\left(\mathcal{L}_{0}(\dot{\boldsymbol{x}}(t))-V_{t}(\boldsymbol{x}(t))\right) .\right.
$$

Ignoring mathematical subtleties, $\mathscr{D} \boldsymbol{x}$ denotes the measure on the family of orbits (paths) $\boldsymbol{x}$, starting at time $t_{i}$ at $\boldsymbol{q}$ and ending at time $t_{f}$ at $\boldsymbol{q}^{\prime}$. Still at a heuristic level, let us assume that this measure is invariant under deformations of the orbits by loops $t \mapsto \boldsymbol{x}_{0}(t)$ about 0 , i.e. smooth functions having their supports in the time interval $t_{i} \leq t \leq t_{f}$. The integrand is thereby transformed into

$$
\mathcal{L}_{0}\left(\dot{\boldsymbol{x}}(t)+\dot{\boldsymbol{x}}_{0}(t)\right)-V_{t}\left(\boldsymbol{x}(t)+\boldsymbol{x}_{0}(t)\right)=\mathcal{L}_{0}(\dot{\boldsymbol{x}}(t))+\delta \mathcal{L}_{0}\left(\dot{\boldsymbol{x}}_{0}(t)\right)(\dot{\boldsymbol{x}}(t))-V_{t}^{\boldsymbol{x}_{0}}(\boldsymbol{x}(t)),
$$

where we make use of the short hand notation 


$$
\begin{aligned}
\delta \mathcal{L}_{0}\left(\dot{\boldsymbol{x}}_{0}\right)(\dot{\boldsymbol{x}}) & :=\mathcal{L}_{0}\left(\dot{\boldsymbol{x}}+\dot{\boldsymbol{x}}_{0}\right)-\mathcal{L}_{0}(\dot{\boldsymbol{x}})=\dot{\boldsymbol{x}_{0}} \dot{\boldsymbol{x}}+(1 / 2) \dot{\boldsymbol{x}}_{0}^{2} \\
V_{t}^{\boldsymbol{x}_{0}}(\boldsymbol{x}) & :=V_{t}\left(\boldsymbol{x}+\boldsymbol{x}_{0}\right) .
\end{aligned}
$$

Now by a partial integration, the velocity $\dot{x}$ in the integral $\int_{t_{i}}^{t_{f}} d t \delta \mathcal{L}_{0}\left(\dot{\boldsymbol{x}}_{0}(t)\right)(\dot{\boldsymbol{x}}(t))$ can be transformed into $\boldsymbol{x}$, so the term $\int_{t_{i}}^{t_{f}} d t\left(\delta \mathcal{L}_{0}\left(\dot{\boldsymbol{x}}_{0}(t)\right)(\dot{\boldsymbol{x}}(t))-V_{t}^{\boldsymbol{x}_{0}}(\boldsymbol{x}(t))\right.$ describes just another perturbation. Plugging this information into the scattering matrix for given perturbing functional $F$ and any given loop $x_{0}$, and putting $F^{x_{0}}[x]:=F\left[x+x_{0}\right]$, we arrive at the equality

$$
S(F)=S\left(F^{x_{0}}+\delta \mathcal{L}_{0}\left(\dot{x}_{0}\right)\right),
$$

containing dynamical information. The second fundamental ingredient in the functional integral formalism is the time ordering involved in the definition of the scattering operators. A perturbing functional $F_{1}$ is said to lie in the future of $F_{2}$ if the underlying potential $t \mapsto V_{1, t}$ in $F_{1}$ has support in the future of $t \mapsto V_{2, t}$, entering in $F_{2}$. The preceding discussion then implies that the resulting scattering matrices satisfy the equality $S\left(F_{1}\right) S\left(F_{2}\right)=S\left(F_{1}+F_{2}\right)$. As a matter of fact, given any other functional $F_{3}$, one obtains by splitting it into $F_{3}=F_{3+}+F_{3-}$, such that $F_{1}+F_{3+}$ lies in the future of $F_{2}+F_{3-}$, the more refined equality [2]

$$
S\left(F_{1}+F_{2}+F_{3}\right)=S\left(F_{1}+F_{3}\right) S\left(F_{3}\right)^{-1} S\left(F_{2}+F_{3}\right) .
$$

The preceding two equalities is all what is needed in order to define dynamical algebras.

\section{Dynamical Algebras}

We leave now the mathematically subtle territory of path integrals and take a fresh look at the problem of describing our interventions into the quantum world. From a macroscopic point of view there are two obvious facts. First, quantum systems are tested by perturbing them; in laboratories this happens in a systematic manner, but its surroundings produce such perturbations as well. We therefore propose to take the operations, induced by perturbations, as primary ingredients of the theory. If one happens to know the particular shape of some perturbation in space and time, being described by a functional $F$, the resulting operation is denoted by $S(F)$. The second ingredient taken from the macroscopic world is the arrow of time. It enters into the quantum world since we can firmly state whether some operation $S\left(F_{1}\right)$ was performed after or before some other operation $S\left(F_{2}\right)$. Moreover, it is impossible to make up for missed operations in the past, time is directed.

Since operations can be performed time and again, it is meaningful to assume that they form a semigroup. As a matter of fact, dealing with experimentally accessible systems, it is also plausible that the effect of some operation can be rubbed out by another one, in accordance with the fact that experiments can be repeated. We therefore postulate that the operations $S(F)$, which can be performed on quantum systems, form a group. It becomes a dynamical group for given Lagrangean. For the 
case at hand of a mechanical system, we take for simplicity the free Lagrangean $\mathcal{L}_{0}$ and rely on findings obtained in the preceding step.

Definition Let $\mathcal{L}_{0}$ be a Lagrangean. An operation, fixed by a functional $F$ describing some perturbation of the dynamics, is represented by the symbol $S(F)$. So, in particular, $S(0)=1$. These symbols generate a dynamical group $\mathcal{G}_{\mathcal{L}_{0}}$. They satisfy the relations

(i) $S(F)=S\left(F^{x_{0}}+\delta \mathcal{L}_{0}\left(\dot{\boldsymbol{x}}_{0}\right)\right)$ for any functional $F$ and loop $\boldsymbol{x}_{0}$

(ii) $S\left(F_{1}+F_{2}+F_{3}\right)=S\left(F_{1}+F_{3}\right) S\left(F_{3}\right)^{-1} S\left(F_{2}+F_{3}\right)$ for any functional $F_{3}$, provided $F_{1}$ lies in the future of $F_{2}$.

Based only on macroscopic (classical) concepts, the dynamical group is defined by these relations; there is no quantization condition built in from the outset. Nevertheless, the group is inherently non-commutative. As we shall see, the value of Planck's constant is determined by operations corresponding to the constant functionals $\boldsymbol{x} \mapsto F_{\mathrm{h}}[\boldsymbol{x}]:=\mathrm{h}, \mathrm{h} \in \mathbb{R}$. These functionals have empty support in time; phrased differently, they can be realized by time integrals $\int d t h(t)=\mathrm{h}$, where $t \mapsto h(t)$ has arbitrary support. It therefore follows from the causality condition (ii) that $S(F) S\left(F_{\mathrm{h}}\right)=S\left(F+F_{\mathrm{h}}\right)=S\left(F_{\mathrm{h}}\right) S(F)$. So the operations $\mathrm{h} \mapsto S\left(F_{\mathrm{h}}\right)$ form an abelian group in the center of $\mathcal{G}_{\mathcal{L}_{0}}$. Without essential loss of generality, we fix a scale and put $S\left(F_{\mathrm{h}}\right)=e^{i \mathrm{~h}} 1, \mathrm{~h} \in \mathbb{R}$. We also note that the dynamical relation (i) implies $S\left(\delta \mathcal{L}_{0}\left(\dot{\boldsymbol{x}}_{0}\right)\right)=1$ for all loops; these equalities correspond to the classical Euler-Lagrange equation.

Having defined the group $\mathcal{G}_{\mathcal{L}_{0}}$, it is a standard procedure to construct a corresponding dynamical algebra $\mathcal{A}_{\mathcal{L}_{0}}$. One declares that the elements $S_{k}$ of the group are basis elements of some complex vector space, leading to finite sums with complex coefficients, $\sum_{k} c_{k} S_{k}$. The adjoint operators are defined by $\left(\sum_{k} c_{k} S_{k}\right)^{*}:=\sum_{k} \bar{c}_{k} S_{k}^{-1}$, where the bar denotes complex conjugation. The rules for multiplication are inherited from the group by the distributive law. One can show [2] that the algebra $\mathcal{A}_{\mathcal{L}_{0}}$ is equipped with a norm which promotes it to a $\mathrm{C}^{*}$-algebra, i.e. an algebra which can be realized as a norm closed subalgebra of the algebra of bounded operators on some Hilbert space.

Before showing that this algebra contains the entire information incorporated in the conventional quantum mechanical setting, let us emphasize that our scheme of constructing dynamical algebras covers a large set of systems of physical interest. What is needed is that the system can be described in terms of some classical configuration space, on which a group acts (the loops in the present case), and some Lagrangean. Moreover, one needs a causal structure, given in the present context by the direction of time. In [1] this scheme was applied to an interacting scalar quantum field in Minkowski space for which one also has a classical configuration space, the analogue of loops, and Lagrangeans. The causal structure, however, has to be replaced by the relativistic one, and is concretely given by the partially ordered set of forward lightcones. With that modification, the definition of the dynamical algebra is identical to the preceding one and one arrives in this manner at a consistent description of field theoretic models, satisfying all fundamental postulates of relativistic quantum field theory. 


\section{Recovery of Quantum Mechanics}

In this section we give a brief account of results established in [2], showing that the dynamical algebra of mechanical systems entails the standard formalism of quantum mechanics. In view of the fact that no explicit quantization condition was incorporated into the dynamical algebra, we are led to the conclusion that the origin of quantum effects is the arrow of time, being encoded in the operations. The specific form of commutation relations then follows from the Lagrangean.

Proceeding from the non-interacting Lagrangean $\mathcal{L}_{0}$, we consider the simplest functionals (perturbations) of the form $F_{f}[\boldsymbol{x}]=\int d t \boldsymbol{f}(t) \boldsymbol{x}(t)+\mathrm{h}$. Here $t \mapsto \boldsymbol{f}(t)$ is some continuous function, the integral extends over the (compact) support of $\boldsymbol{f}$, and $\mathrm{h}$ is a constant. A convenient choice which simplifies subsequent formulas is

$$
\mathrm{h}(\boldsymbol{f}):=(1 / 2) \iint d s d s^{\prime}\left|s-s^{\prime}\right| \boldsymbol{f}(s) \boldsymbol{f}\left(s^{\prime}\right)
$$

Given another function $\boldsymbol{f}^{\prime}$ for which the first two moments coincide with those of $\boldsymbol{f}$, i.e.

$$
\int d t\left(\boldsymbol{f}^{\prime}(t)-\boldsymbol{f}(t)\right)=\int d t t\left(\boldsymbol{f}^{\prime}(t)-\boldsymbol{f}(t)\right)=0,
$$

their difference $\ddot{\boldsymbol{x}}_{0}=\boldsymbol{f}^{\prime}-\boldsymbol{f}$ determines a loop function

$$
t \mapsto \boldsymbol{x}_{0}(t)=\int_{-\infty}^{t} d s(t-s)\left(\boldsymbol{f}^{\prime}-\boldsymbol{f}\right)(s) .
$$

By a straightforward computation one obtains for the resulting functionals the equalities, cf. [2],

$$
F_{f}[x]=F_{f^{\prime}}\left[x+x_{0}\right]+F_{-\ddot{x}_{0}}[x]=F_{f^{\prime}}^{x_{0}}[x]+\delta \mathcal{L}_{0}\left(\dot{x}_{0}\right)[x]
$$

Plugging the latter expression into the dynamical relation (i), one arrives at $S\left(F_{f}\right)=S\left(F_{f^{\prime}}\right)$ for arbitrary pairs of functions $f, f^{\prime}$ whose first two moments coincide. In particular, $S\left(F_{\ddot{x}_{0}}\right)=1$ for all loop functions $\boldsymbol{x}_{0}$. As we shall see, these relations determine solutions of the Heisenberg equation.

Next, let $F_{\boldsymbol{g}}[\boldsymbol{x}]=\int d t \boldsymbol{g}(t) \boldsymbol{x}(t)+\mathrm{h}(\boldsymbol{g})$ be another functional. We want to determine the relation between the unitary operators $S\left(F_{f}\right)$ and $S\left(F_{g}\right)$. To this end we replace $f$ as in the preceding step by a function $\boldsymbol{f}^{\prime}$ which has support in the future of $\boldsymbol{g}$. Making use of the causal relation (ii), we obtain

$$
S\left(F_{f}\right) S\left(F_{g}\right)=S\left(F_{f^{\prime}}\right) S\left(F_{g}\right)=S\left(F_{f^{\prime}}+F_{g}\right) .
$$

In view of the linear dependence of the given functionals on the underlying orbits it is apparent that $F_{f^{\prime}}+F_{g}-F_{f^{\prime}+g}$ is a constant functional. By another routine computation one finds [2]

$$
F_{f^{\prime}}[x]+F_{g}[x]-F_{f^{\prime}+g}[x]=-(1 / 2)\left\langle f^{\prime}, \Delta g\right\rangle,
$$


where we have defined

$$
\left\langle\boldsymbol{f}^{\prime}, \Delta \boldsymbol{g}\right\rangle:=\iint d s d s^{\prime} \boldsymbol{f}^{\prime}(s)\left(s^{\prime}-s\right) \boldsymbol{g}\left(s^{\prime}\right) .
$$

In view of the fact that this is a constant functional on the space of paths, we can proceed to

$$
S\left(F_{f^{\prime}}+F_{g}\right)=S\left(F_{f^{\prime}+g}-(1 / 2)\left\langle f^{\prime}, \Delta \boldsymbol{g}\right\rangle\right)=S\left(F_{f^{\prime}+g}\right) e^{-(i / 2)\left\langle f^{\prime}, \Delta g\right\rangle},
$$

where in the second equality our choice of scale for the constant functionals entered. Now, by construction, the first and second moments of the functions $\left(\boldsymbol{f}^{\prime}+\boldsymbol{g}\right)$ and $(f+g)$ coincide, so according to the first step we have $S\left(F_{f^{\prime}+g}\right)=S\left(F_{f+g}\right)$. By inspection of its defining equation, it is also clear that $\left\langle\boldsymbol{f}^{\prime}, \Delta \boldsymbol{g}\right\rangle=\langle\boldsymbol{f}, \Delta \boldsymbol{g}\rangle$. Thus we have arrived at the equality

$$
S\left(F_{f}\right) S\left(F_{g}\right)=e^{-(i / 2)\langle f, \Delta g\rangle} S\left(F_{f+g}\right) .
$$

This relation shows that the unitary operators $S\left(F_{f}\right)$ do not commute amongst each other. They form a familiar Lie group, the Weyl group. We proceed from this Lie group to its Lie algebra (defined in suitable representations) and represent the unitaries by exponentials of the corresponding generators,

$$
e^{i \int d t \boldsymbol{f}(t) \boldsymbol{Q}(t)}:=S\left(F_{f}\right) .
$$

Now, as we have seen, $S\left(F_{\ddot{x}_{0}}\right)=S(0)=1$ for the second derivatives of all loop functions $\boldsymbol{x}_{0}$. So the time dependence of the generators is given by

$$
t \mapsto \boldsymbol{Q}(t)=\boldsymbol{Q}+t \dot{\boldsymbol{Q}} .
$$

Putting $\boldsymbol{a}:=\int d t \boldsymbol{f}(t)$ and $\boldsymbol{b}:=\int d t t \boldsymbol{f}(t)$, we therefore obtain

$$
\int d t \boldsymbol{f}(t) \boldsymbol{Q}(t)=\boldsymbol{a} \boldsymbol{Q}+\boldsymbol{b} \dot{\boldsymbol{Q}}=\sum_{k=1}^{3 N}\left(\boldsymbol{a}_{k} \boldsymbol{Q}_{k}+\boldsymbol{b}_{k} \dot{\boldsymbol{Q}}_{k}\right)
$$

In view of the freedom to choose the functions $f$, it then follows from the above Weyl relations for the exponentials that

$$
\left[\boldsymbol{Q}_{k}, \dot{\boldsymbol{Q}}_{l}\right]=i \delta_{k l} 1, \quad\left[\boldsymbol{Q}_{k}, \boldsymbol{Q}_{l}\right]=\left[\dot{\boldsymbol{Q}}_{k}, \dot{\boldsymbol{Q}}_{l}\right]=0
$$

Identifying $\boldsymbol{Q}$ with the observable "position" and $\dot{\boldsymbol{Q}}$ with "momentum", we have thus arrived at the Heisenberg commutation relations. With our choice of scale for the constant functionals, Planck's constant is put equal to 1 (atomic units). We also find that the time evolution of the operators is given by the solution of the Heisenberg equation $\dot{\boldsymbol{Q}}(t)=i\left[H_{0}, \boldsymbol{Q}(t)\right]$, where $H_{0}=(1 / 2) \dot{\boldsymbol{Q}}^{2}$ is the Hamiltonian corresponding to the underlying Lagrangean. So we have recovered from our macroscopic point of view the structure of quantum mechanics.

Interacting systems of particles can now be described by making use of an abstract version of the interaction picture. Consider a Lagrangean of the form 


$$
\mathcal{L}(\boldsymbol{x}, \dot{\boldsymbol{x}})=(1 / 2) \dot{\boldsymbol{x}}^{2}-V_{I}(\boldsymbol{x})
$$

where $V_{I}$ describes the interaction. One first localizes it in time by some characteristic function $t \mapsto \chi(t)$ with arbitrary compact support. This yields the time-dependent Lagrangean

$$
\mathcal{L}_{\chi}(t, \boldsymbol{x}, \dot{\boldsymbol{x}})=(1 / 2) \dot{\boldsymbol{x}}^{2}-\chi(t) V_{I}(\boldsymbol{x})
$$

Defining $\chi V_{I}[\boldsymbol{x}]:=\int d t \chi(t) V_{I}(\boldsymbol{x}(t))$, one then introduces relative operations $S_{\mathcal{L}_{\chi}}$. They depend on functionals $F$ as in the non-interacting case which are given by

$$
S_{\mathcal{L}_{\chi}}(F):=S\left(-\chi V_{I}\right)^{-1} S\left(F-\chi V_{I}\right)
$$

Here $S$ are the operations considered before in the non-interacting case. Thus the operators $S_{\mathcal{L}_{\chi}}(F)$ are still elements of the non-interacting algebra $\mathcal{A}_{\mathcal{L}_{0}}$. Moreover, by some standard computation [2] one finds that they satisfy the defining relations for the dynamical algebra $\mathcal{A}_{\mathcal{L}_{\chi}}$, where the non-interacting Lagrangean $\mathcal{L}_{0}$ in the definition given in Sect. 3 is replaced by $\mathcal{L}_{\chi}$. So all these dynamical algebras can be accommodated in $\mathcal{A}_{\mathcal{L}_{0}}$. By some more detailed analysis [2], one then shows that this feature prevails for the algebra $\mathcal{A}_{\mathcal{L}}$, which is obtained in the limit $\chi \rightarrow 1$. To be precise, this has been established so far only for some large family of interaction potentials. Condoning this point, we conclude that there is a single dynamical algebra for all mechanical systems, the algebra $\mathcal{A}_{\mathcal{L}_{0}}$. Algebras corresponding to different Lagrangeans merely amount to a reinterpretation of its elements.

We conclude this section by noting how one can proceed from the present abstract algebraic setting to the familiar Hilbert space formulation in the Schrödinger representation, based on wave functions $\boldsymbol{x} \mapsto \psi(\boldsymbol{x})$. There one represents $\boldsymbol{Q}$ by multiplying the wave functions with $\boldsymbol{x}$ and $\boldsymbol{P}$ by taking their gradient $-i \partial_{x}$. The operators $S(F)$, representing operations, are then given by time ordered exponentials,

$$
S(F) \simeq T e^{i \int d t F(\boldsymbol{Q}+t \boldsymbol{P})} .
$$

We also note that the familiar statistical interpretation of quantum mechanics in terms of expectation values of observables can be based on the present concept of operations. The interested reader may consult [2] for further details.

\section{Summary and Outlook}

In the present letter we have established a relation between the path integral approach to quantum physics and the framework of dynamical algebras, established in $[1,2]$. This new approach is based on a macroscopic concept of operations, describing perturbations of the dynamics; it dispenses with assumptions about their actual implementation in the quantum world. Quantum effects are inherited from the macroscopic arrow of time, determining the causal structure of operations; detailed properties, such as commutation relations, then follow from the given dynamics. 
The framework of dynamical algebras avoids the mathematical subtleties involved in the definition of path integrals; but it comprises the same physical information. It is defined in the mathematically well established and convenient setting of $\mathrm{C}^{*}$ algebras. As we have shown, computations of integrals can be replaced by algebraic manipulations.

Such as the path integral formalism, the framework of dynamical algebras covers a large class of theories. It requires that classical concepts, such as the notion of configuration space, transformation groups, Lagrangeans and macroscopic causal structures provide a physically meaningful starting point. With this input, it offers a complementary approach to quantum physics and already led to progress in the rigorous formulation of interacting quantum field theories [1]. Within that context, it seems worthwhile to have a look at other longstanding problems. Examples are an algebraic version of Noether's theorem and the study of anomalies; the algebraic treatment of gauge quantum field theories and an algebraic version of the renormalization group. For the mathematical consolidation of quantum field theory in physical spacetime it would, however, be most important to make progress in the representation theory of dynamical algebras which goes beyond renormalized perturbation theory.

Acknowledgements Open Access funding provided by Project DEAL.

Open Access This article is licensed under a Creative Commons Attribution 4.0 International License, which permits use, sharing, adaptation, distribution and reproduction in any medium or format, as long as you give appropriate credit to the original author(s) and the source, provide a link to the Creative Commons licence, and indicate if changes were made. The images or other third party material in this article are included in the article's Creative Commons licence, unless indicated otherwise in a credit line to the material. If material is not included in the article's Creative Commons licence and your intended use is not permitted by statutory regulation or exceeds the permitted use, you will need to obtain permission directly from the copyright holder. To view a copy of this licence, visit http://creativecommons.org/licen ses/by/4.0/.

\section{References}

1. Buchholz, D., Fredenhagen, K.: A $\mathrm{C}^{*}$-algebraic approach to interacting quantum field theories. arXiv:1902.06062. Commun. Math. Phys. (2020). https://doi.org/10.1007/s00220-020-03700-9

2. Buchholz, D., Fredenhagen, K.: Classical Dynamics, Arrow of Time, and Genesis of the Heisenberg Commutation Relations (2020). arXiv:1905.02711

3. Dirac, P.A.M.: The Lagrangian in quantum mechanics. Phys. Z. Sowjetunion 3, 64-72 (1933)

4. Feynman, R.P., Hibbs, A.R.: Quantum Mechanics and Path Integrals. McGraw-Hill, New York (1965)

Publisher's Note Springer Nature remains neutral with regard to jurisdictional claims in published maps and institutional affiliations. 